\title{
Polymeric Nanostructures as Colloidal Drug Delivery Systems: Thermosensitive Hydrogels Containing Self-Assembled Micelles
}

Mohammad Nasri ${ }^{1 *}$ and Hossein Mirshekarpour ${ }^{2}$

${ }^{1}$ Afzalipour School of Medicine, Kerman University of Medical Sciences and Health Services, Kerman, Iran

${ }^{2}$ Nuclear Medicine Department of Shafah Hospital, Kerman University of Medical Sciences and Health Services, Kerman, Iran

\begin{abstract}
Polymeric nanostructures for drug delivery applications including smart hydrogels and stealth micelles have been developed to overcome many obstacles in the way of timing and targeting the delivery to fulfill therapeutic potentiality of chemicals. The purpose of this investigation is to prepare and characterize novel nanoparticle-based colloidal products composed of biocompatible polymers to use for rate-controlled release and targeted/spatial drug delivery systems, aiming specifically at drug delivery via mucosal routes. We formulated the new products with inert and biocompatible polymers including sodium carboxymethylcellulose $(\mathrm{NaCMC})$ and polysorbates. The synthesis process was performed by heating/cooling method, as a type of physical cross-linking method for producing hydrogel, in three steps to optimize physical and chemical characteristics of the products to make them suitable for delivery through mucosal routes. A series of nanoparticle-based colloidal products in the form of liquid suspensions were developed, and from all three steps, thirty-nine samples were selected and rheologically investigated by bench-top experiments. A freeze-thawing method was applied to two samples of product III10 for five and one repeated cycles sequentially. Test tube inversion method was also carried out on all the produced gels. Most of the products are new types of temperaturesensitive, smart, physically self-assembled hydrogels and took the forms of sol, gel (opaque and transparent), and precipitate. However, these hydrogels show opposite gelation property to customary temperature-sensitive gels. The product of five repeated freezing-thawing cycles is also thermoreversible gel with a high mechanical stability and swelling capacity, as opposed to the product of one cycle. Some types of produced hydrogels behave like a ternary system. The products of this work, with microstructure consisting of the polymeric chains of sodium carboxymethylcellulose and a large-scale self-assembly of micellar structures of polysorbates incorporated within a polymer network, show major efficacy of site-specific and controlled release drug delivery system.
\end{abstract}

Keywords: Drug delivery; Polymeric nanostructure; Temperature sensitive smart hydrogel; Micelle; Self-assembly; Mucosal drug delivery; Mucoadhesion

\section{Introduction}

An extraordinary effort, over recent decades, has yielded a great achievement of novel chemicals with a revolutionary potential as therapeutics [1-4]. However, their potential has hit practical problems because of many obstacles in the way of their delivery in the body [3]. The chemicals need to get to the right place at the right time to be effective, and thus drug delivery and targeting was taken into consideration [3]. Advanced drug delivery and targeting has seen spectacular achievements [1-4] including the development of a number of novel delivery systems and state-of-the-art technologies by researchers from a wide range of disciplines. More recently, utilizing the polymer science in modern-day pharmaceutical science and technology, concurrent with a dramatic increase in the types of available polymers, has started a technological revolution in drug delivery systems and culminated in controlled drug release and long-circulating pharmaceutical carriers [4-7]. It is found that [8-10] nanocarriers composed of biocompatible materials can be used as alternatives to current delivery systems. Nanoparticles as drug delivery systems can passively target the drug preferentially to intracellular targets, tumors, and inflammation sites by enhanced permeability and retention effect (EPR) $[8,11,12]$. Using biodegradable polymers in formulating nanoparticles brings sustained drug release about [11]. Additionally, surface modification of nanoparticles with hydrophilic polymers/surfactants such as polyethylene glycol (PEG), polyethylene oxide (PEO), and polysorbate 80 decreases opsonization and causes prolonged circulation time $[11,12]$. To minimize amount of polymer matrix of delivery system, nanodelivery system needs to have a high drug loading capacity [11].
By developing new types of polymer-based colloidal drug delivery systems such as micelles [13], liposomes [5], nano- and microparticles $[5,12]$ and chemical and physical hydrogels $[14,15]$, if capability of the stimulus-responsive phase separation was provided, timing and targeting the delivery can be developed $[7,12,16]$. With the intention of inventing biomimetic systems capable of drug release following biological need, hydrogel-based drug delivery systems $[4,6,14,15,17]$ and stimuli-sensitive intelligent hydrogels [18-20] have been developed. The types of physically self-assembled hydrogels, in which the microstructure is made up of arranged micelles, are taking an increasingly prominent part in drug delivery and controlled release $[3,4,7,14]$. It is known those types of micelles which formed by aggregation of nonionic surfactants are the most commonly employed micelles in controlled release delivery systems [21]. Physical mixtures of polymers involving polymer blends or interpenetrating networks $[22,23]$ open up ample opportunity as desired delivery systems.

Several studies have been performed to prepare more efficacious polymeric nanostructure based systems for controlled and targeted

*Corresponding author: Mohammad Nasri, Afzalipour School of Medicine, Kerman University of Medical Sciences and Health Services, Pajouhesh Sq., Bistodo Bahman Blvd., Kerman, Iran, Tel 98-910-6879305; E-mail: mohammadnasri@kmu. ac.ir, mnasri.bme@gmail.com

Received April 17, 2015; Accepted May 11, 2015; Published June 04, 2015

Citation: Nasri M, Mirshekarpour H (2015) Polymeric Nanostructures as Colloidal Drug Delivery Systems: Thermosensitive Hydrogels Containing Self-Assembled Micelles. J Nanomed Nanotechnol 6: 301. doi:10.4172/2157-7439.1000301

Copyright: @ 2015 Nasri M, et al. This is an open-access article distributed under the terms of the Creative Commons Attribution License, which permits unrestricted use, distribution, and reproduction in any medium, provided the original author and source are credited. 
drug delivery $[8,24]$. The aim of the present work is to develop smart hydrogel-nanoparticles colloidal products in order to employ as new drug delivery and release systems to overcome the obstacles regarding timing and targeting the delivery, and to use these systems for ratecontrolled release and targeted/spatial drug delivery, with special aim of mucosal drug delivery. To succeed in facing up to these considerations, we have formulated and synthesized a series of thermally sensitive hydrogels in the form of liquid suspensions, which based on physically blended polymer matrices of sodium carboxymethylcellulose (NaCMC) and polysorbates (tweens). The temperature sensitivity of these gels are explored by rheological experiments and test tube inversion method. In order for these delivery systems to use for mucosal delivery, in addition to mucoadhesion characteristics of the delivery systems, physical and chemical properties of these liquid suspensions are optimized by adjusting the concentration of $\mathrm{NaCMC}$, tweens and glycerin [25].

\section{Material and Methods}

\section{Materials}

Sodium carboxymethylcellulose, some types of polysorbates (tweens) including polysorbates 80, 20, 40,60, and 65, and glycerol (glycyl alcohol) were all purchased from Merck KGaA (Darmstadt, Germany) and used as received without further purification.

$\mathrm{NaCMC}$ is an inert and biocompatible polymer and mostly accepted as a non-toxic material [2]. Essentially, NaCMC, because of great capability to absorb and react with water and moisture, can form viscoelastic gum [26,27]. Polysorbates, and more especially polysorbate 80 , have been most commonly used $[21,28]$ among the micelle forming amphiphilic molecules. Polysorbates have generally been acknowledged as non-toxic materials because of their relatively low toxicities and inert nature $[2,29,30]$. Glycerol is widely used in pharmaceutical formulations as a co-solvent and also to pre-wet the powder, and is generally held to be a non-toxic material, with minimum adverse effects because of its dehydrating property $[2,29,30]$.

\section{Preparation of physical cross-linking hydrogels}

Heating/cooling method: Given that cellulose gum goes rapidly into the solution, and afterwards, when dispersed into water will swell to sticky gel grains and most likely form lumps, it is first necessary to disperse and wet NaCMC powder in glycerin as a co-solvent water miscible liquid that prevents $\mathrm{NaCMC}$ from swelling. The slurry is then added to water, and a lump-free clear solution is prepared [31].

The experiments were implemented in a beaker equipped with a mechanical stirrer. Firstly, at room temperature a preordained amount of glycerin was exactly weighed into the beaker, and then the $\mathrm{NaCMC}$ with prescribed concentration was sprinkled onto the glycerin and stirred gently for ten minutes until all its particles were completely wet. The slurry was then slowly added into various chosen amounts of deionized water to obtain the solutions with given compositions, and the mixture was then heated with moderate shear to about $60^{\circ} \mathrm{C}$ and mixed to have the gum completely hydrated and obtain a homogenous blended solution. The mixture was then cooled to $45^{\circ} \mathrm{C}$, and thereafter the polysorbates, under the given amounts of specified types, was added. Mixing was continued while cooling the mixture to $30^{\circ} \mathrm{C}$. The polymer mixture was left to room temperature overnight leading to the complete formation of a thermally sensitive hydrogel.

Optimization of properties: Concerning the physicochemical properties of novel products, the formulation and synthesis process was executed in three steps: (1) optimizing the viscosity and, more especially, the kinematic viscosity; (2) lowering the surface tension; and (3) optimizing all selected properties [25]. In the first step, with the aim of optimizing the viscosity, only the concentration of $\mathrm{NaCMC}$ among the ingredients was taken into consideration and considered variable (Table 1). And on the other hand, for this step, the concentrations of polysorbate 80 and glycerol were considered fixed and about $0.3 \%$ and $30 \mathrm{~cm}^{3}$ correspondingly.

Next, the experiments were focused on lowering the surface tension. To this aim, the amounts of glycerin and NaCMC were considered almost fixed and about $30 \mathrm{~cm}^{3}$ and 0.7 gr respectively, and then various concentrations of tween 80 started with $1 \%(\mathrm{w} / \mathrm{w})$ were utilized to adjust the surface tension values. In the final step, to synchronously optimize and achieve the desired amounts of viscosity (dynamic and kinematic), density and surface tension in a product type, polysorbate 20 and some other types such as polysorbates 60 and 65 and finally polysorbate 40 , along with other ingredients, were utilized in varied concentrations (Table 2). Viscosity was measured using a Programmable Rheometer (Brookfield, DV.III, ULTRA, Engineering Laboratories, INC., Middleboro, USA) and surface tension was measured using a KRUSS Tensiometer (model K100C, Germany).

Freeze-thawing method: Product type $\mathrm{III}_{10}$ was used to prepare an aqueous solution by heating the colloidal product to about $60^{\circ} \mathrm{C}$ under stirring until it was completely homogeneous and an aqueous solution was obtained. Thereafter, two samples of this aqueous solution were placed in a freezer at $-20^{\circ} \mathrm{C}$ and maintained for 24 hours. After the freezing process, frozen samples were allowed to thaw at $25^{\circ} \mathrm{C}$ for 24 hours. This freezing and thawing method was repeated for four more cycles for one of the above samples, but the other sample was left with no further additional process. Afterwards, all the samples were stored at $4^{\circ} \mathrm{C}$.

\begin{tabular}{|c|c|c|c|}
\hline Suspension type & $\mathrm{I}_{1}$ & $\mathrm{I}_{2}$ & $\mathrm{I}_{3}$ \\
\hline NaCMC & $0.5 \%$ & $0.75 \%$ & $0.85 \%$ \\
\hline
\end{tabular}

Table 1: NaCMC values in the selected suspensions of the first step of formulation.

\begin{tabular}{|c|c|c|c|}
\hline Suspension type & NaCMC (gr) & Gly $\left(\mathrm{cm}^{3}\right)$ & Tweens \\
\hline $\mathrm{III}_{1}$ & 1.5 & 30 & 20 \\
\hline $\mathrm{III}_{2}$ & 1 & 30 & $\begin{array}{l}2 \% \\
20\end{array}$ \\
\hline \multirow{2}{*}{$\mathrm{III}_{3}$} & \multirow{2}{*}{0.7} & \multirow{2}{*}{30} & 20 \\
\hline & & & $2 \%$ \\
\hline \multirow{2}{*}{$\mathrm{III}_{4}$} & \multirow{2}{*}{0.7} & \multirow{2}{*}{25} & 20 \\
\hline & & & $2 \%$ \\
\hline \multirow{2}{*}{$\mathrm{III}_{5}$} & \multirow{2}{*}{0.7} & \multirow{2}{*}{30} & 60 \\
\hline & & & $2 \%$ \\
\hline \multirow{2}{*}{$\mathrm{III}_{6}$} & \multirow{2}{*}{1} & \multirow{2}{*}{30} & 60 \\
\hline & & & $2 \%$ \\
\hline \multirow{2}{*}{$\mathrm{III}_{7}$} & \multirow{2}{*}{1} & \multirow{2}{*}{25} & 60 \\
\hline & & & $2 \%$ \\
\hline \multirow{2}{*}{$\mathrm{III}_{8}$} & \multirow{2}{*}{1.5} & \multirow{2}{*}{30} & 65 \\
\hline & & & $2 \mathrm{gr}$ \\
\hline \multirow{2}{*}{$\mathrm{III}_{9}$} & \multirow{2}{*}{1} & \multirow{2}{*}{30} & 40 \\
\hline & & & $5 \%$ \\
\hline \multirow{2}{*}{$\mathrm{III}_{10}$} & \multirow{2}{*}{1.5} & \multirow{2}{*}{30} & 65 \\
\hline & & & $2 \%$ \\
\hline
\end{tabular}

Table 2: Compositions of ingredients in some selected samples of final step of formulation. 
Test tube inversion method: Thermo-responsiveness, and swelling and shrinking behaviors of the polymer gels were experimented by a test tube inversion method. The sol (flow)-gel (no flow) phase transformation was determined by warming up the stored samples at $4^{\circ} \mathrm{C}$. The sample was considered to be in the gel state when no visual flow was observed in $30 \mathrm{~s}$ after inverting the vial, and in the sol state if it flowed.

\section{Rheological characterization (Bench-top experiments)}

Bench-top experiments were conducted on the selected samples from the three steps of formulation and the products of freeze-thawing method by employing simple and bifurcated glass capillary tube models of airways and catheter combination [25]. Of these, various samples of liquid suspensions that were instilled through a catheter inserted in the capillary tube were rheologically investigated, as well as the effects of different instillation and delivery protocols [25].

\section{Results}

Executing formulation and production, a number of polymeric nanostructure-mediated systems as liquid suspensions were synthesized. The new suspensions comprising thirty-nine types selected from three steps of heating/cooling method and the products of freeze-thawing method were all prepared to employ as the novel polymer-based colloidal drug delivery and release systems, and also as a new group of carriers aiming at mucosal routes of drug delivery. It has been revealed over a long period of time that these products are physically and chemically stable. Actually, most of these new samples including the products of freeze-thawing technique were the new types of temperature-sensitive smart hydrogels, which took amorphous forms and were physically self-assembling polymer nanostructures. All the hydrogel products of this work presented the three essential physical forms including sol, gel, and precipitate. Furthermore, the gels exhibited the quality of an opaque or transparent form. Figure 1 shows optical images of some hydrogel samples of the present study in the forms of sol, transparent gel, opaque gel, and precipitate. All the prepared products in step I were transparent, while the products of step II and III were opaque.

For some types of these produced mixtures, such as $\mathrm{I}_{2}$ and $\mathrm{I}_{3}$, at the end of the swelling process, hydrogel chains dissolved completely in solvents and occupied the entire space of the mixtures, which was occupied by the solvents heretofore (hydrosol). Both types $\mathrm{I}_{2}$ and $\mathrm{I}_{3}$ seemed viscous transparent liquids, among which viscousness of type $I_{3}$ appeared to be much more than $I_{2}$. On the other hand, for type $I_{1}$, which was in a fully swollen state, hydrogel molecules were totally hydrated in considerable amounts and simply dissolved in solvents, and therefore a weak and watery suspension was obtained. A lot of products, such as III $_{1}$ exhibited the pseudo-swelling behavior, which was defined by no real physical swelling and made the transition to polymer network (hydrogel). These types also developed water uptake to a lesser extent and showed relatively higher strength, viscosity, and elasticity, and were difficult to flow.

The thermo-reversibility of the novel hydrogels became pretty obvious from both test-tube inverting experiments and rheological studies, in which the hydrogels underwent sol-gel or gel-sol phase transformation depending on changes in temperature. However, these hydrogels showed the opposite gelation property to customary temperature-sensitive gels in the manner in which temperature increments brought the weaker gels about and dissociated the hydrogel network, and ultimately made the transition process which resulted in solution development (sol state). A higher rise in temperature in prepared products eventually resulted in a precipitate state. Conversely, lowering the temperature brought about a more integrated and condensed gel network. These hydrogels showed reversible switching between swelling and shrinking corresponding to temperature changes. Keeping the produced hydrogels at around $4^{\circ} \mathrm{C}$ induced swelling of the gels in solvent, but contrariwise the gel networks shrank at higher temperatures. Figure 2 shows some visual images of these thermoresponsive hydrogels in the swollen state. The visual images of these polymer gels in the shrinking state are also shown in Figure 3.

The product of five repeated freezing-thawing cycles and some types of the polymer gels developed in the three steps of optimization of properties such as type III $_{10}$ behaved like a ternary system. The ternary system consisted of three phases involving the denser and murkier part of the colloidal suspension, which was placed on the looser and clearer part. The third part, on the other hand, was the swollen gel, which included the dendritic chains (agglomerates) suspended in the denser part. Figure 4 displays some photographs of these ternary systems of product type $\mathrm{III}_{10}$ and product of five repeated freezing-thawing cycles with the three phases.

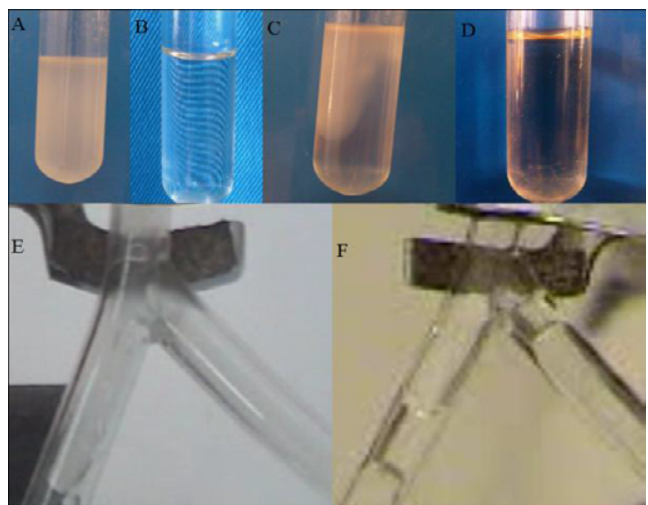

Figure 1: Visual images of the sol, transparent gel, opaque gel, and precipitate states of the produced hydrogels. Two types of sol products (A $B)$, gel (C), and precipitate (D). Opaque gel (E) and transparent gel (F) in bifurcated glass capillary tube models of airways at the time of rheological characterization.

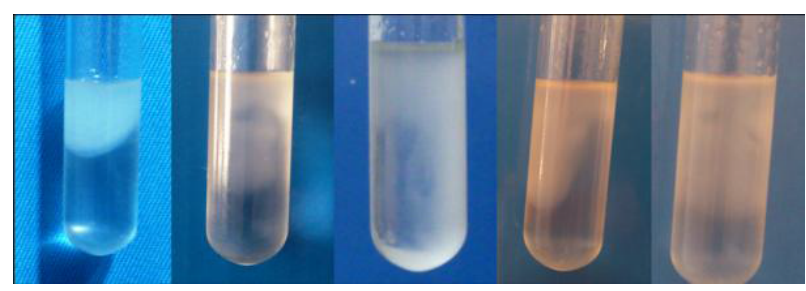

Figure 2: Optical images of some produced temperature-sensitive hydrogels in a swollen state.

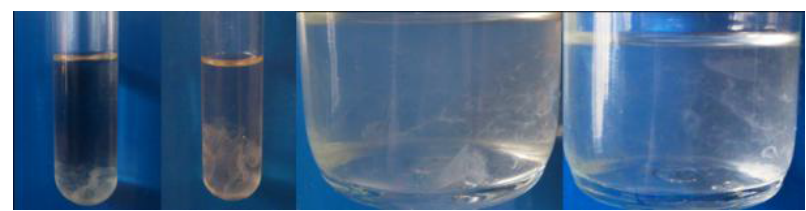

Figure 3: Optical images of some produced thermosensitive polymer hydrogels in a shrinking state. 
The hydrogels produced in the two samples for which the method of freezing-thawing was adopted were markedly different in their characteristics including stability, behavior, nature, and mechanical strength. In the aqueous polymer solution, which was exposed to five repeated cycles, a strong hydrogel with high mechanical integrity was formed. This hydrogel owned some unique properties including significant densification and aggregation, stability at room temperature, high swelling capacity, and high mechanical strength. After incubation and storing, this sample took the form of a ternary system. The produced gel in the sample with one freezing-thawing cycle was not stable enough to preserve its weight and integrity even immediately after the freezing, and it swelled to the full extent.

Optimizing the physicochemical properties of the products in regard to using for mucosal delivery was discussed in detail by Nasri et al. [25], in which these properties were adjusted to employing in mucosal routes, and to the natural amounts of secreted mucus in these routes including airway surface liquid. Among the prepared suspensions in step I, three products were selected regarding their viscosities, which were very different from each other (Table 3) [25]. Carrying out step II, the surface tension amount was lowered [25], and accordingly, twenty-six types of prepared suspensions in this step were

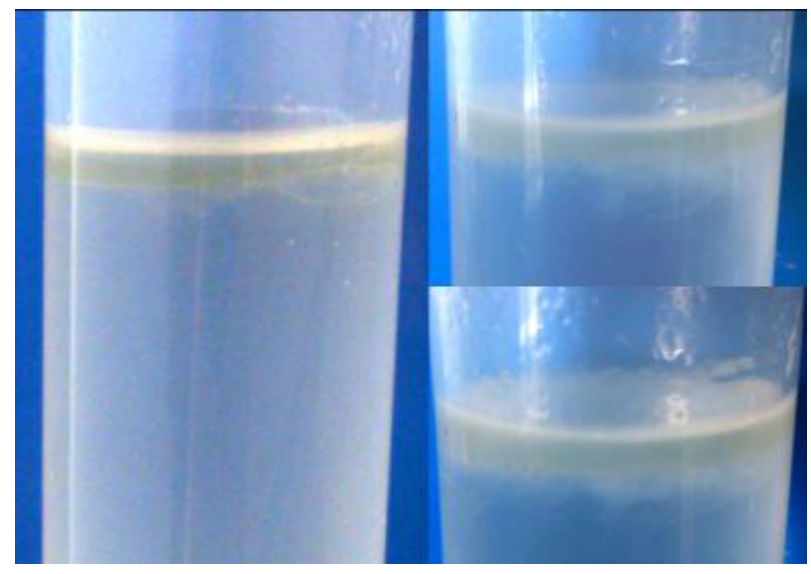

Figure 4: Visual images of the ternary systems consisting of the swollen gel (first phase), which included the dendritic chains (agglomerates) suspended in a high molecular weight solvent (second phase). And the third phase is the looser part of polymer solution.

\begin{tabular}{|c|c|c|c|c|}
\hline $\begin{array}{c}\text { Suspension } \\
\text { type }\end{array}$ & $\begin{array}{l}\text { Vis } \\
\text { (Pa.s) }\end{array}$ & $\begin{array}{c}\text { Den } \\
\left(\mathbf{k g ~ m}^{-3}\right)\end{array}$ & $\begin{array}{c}\text { kVis } \\
\left(m^{2} s^{-1}\right)\end{array}$ & $\begin{array}{c}\text { TS } \\
\left(\mathbf{N ~ m}^{-1}\right)\end{array}$ \\
\hline$I_{1}$ & 0.0175 & 1090 & $0.161 \times 10^{-4}$ & 0.05832 \\
\hline$I_{2}$ & 0.0284 & 1070 & $0.265 \times 10^{-4}$ & 0.06000 \\
\hline$I_{3}$ & 0.0681 & 1060 & $0.642 \times 10^{-4}$ & 0.06300 \\
\hline IIII $_{1}$ & 0.0601 & 1140 & $0.527 \times 10^{-4}$ & 0.03048 \\
\hline $\mathrm{III}_{2}$ & 0.0405 & 1400 & $0.289 \times 10^{-4}$ & 0.03092 \\
\hline $\mathrm{III}_{3}$ & 0.0370 & 1300 & $0.285 \times 10^{-4}$ & 0.03340 \\
\hline $\mathrm{III}_{4}$ & 0.0420 & 1230 & $0.341 \times 10^{-4}$ & 0.02987 \\
\hline $\mathrm{III}_{5}$ & 0.0530 & 1200 & $0.442 \times 10^{-4}$ & 0.02925 \\
\hline $\mathrm{III}_{6}$ & 0.0620 & 1059 & $0.585 \times 10^{-4}$ & 0.02860 \\
\hline $\mathrm{III}_{7}$ & 0.0600 & 1100 & $0.545 \times 10^{-4}$ & 0.02925 \\
\hline $\mathrm{III}_{8}$ & 0.0650 & 1050 & $0.619 \times 10^{-4}$ & 0.02860 \\
\hline $\mathrm{III}_{9}$ & 0.0650 & 1088 & $0.597 \times 10^{-4}$ & 0.02898 \\
\hline $\mathrm{III}_{10}$ & 0.0549 & 1116 & $0.492 \times 10^{-4}$ & 0.02881 \\
\hline
\end{tabular}

Table 3: Amounts of physicochemical characteristics including dynamic viscosity (Vis), kinematic viscosity (kVis), density (Den) and surface tension (TS) of three and ten selected types of suspension products sequentially in step one and three of formulation. selected. In the stage three, ten types among the produced samples were selected. In this step, as represented in Table 3, measures of all the parameters were optimized [25]. Table 3 shows the amounts of viscosity (dynamic and kinematic), density, and surface tension of three and ten selected types from prepared suspensions in the first and final stages of formulation serially. The values of dynamic viscosity (Vis), kinematic viscosity (KVis), density (Den), and surface tension (TS) are presented sequentially in Pa.s, $\mathrm{m}^{2} \mathrm{~s}^{-1}, \mathrm{~kg} \mathrm{~m}^{-3}$, and $\mathrm{N} \mathrm{m}^{-1}$.

\section{Discussion}

Novel drug delivery systems have been highly developed as the latest in some technologies, including: (a) colloidal carriers to control duration of local delivery, and the therapeutic blood and tissue level of drugs aimed at prolonged or sustained release, (b) dispersed systems, ie suspensions and particulate carriers, (c) hydrogel-nanoparticles and smart hydrogels, ie temperature-sensitive ones to provide sustained and controlled release, (d) stealth micelles, (e) bioadhesive polymers, mucoadhesive in this case, to prolong contact time with the mucosal surfaces and improve retention of delivery systems in the mucosal routes with no local harm or adverse effects, and (f) penetration enhancers to safely increase absorption of poorly absorbed moieties with no toxicity during prolonged or sustained delivery. One main issue considered in choosing each and every one of the essential ingredients and also employing various compositions of them was the loading capacity of the delivery systems with chemicals of different solubility states and physicochemical and surface characteristics.

NaCMC was used to synthesize the new types of hydrogel with demanded characteristics. $\mathrm{NaCMC}$, in water, provides high viscosity polyanionic hydrogel (physical or chemical) [23,32] with many excellent properties such as high water content, good biodegradation, excellent biocompatibility, and low-cost yielded advantages for a drug delivery system $[4,18,26]$. Heating-cooling a polymer solution is one of the assorted physical cross-linking methods cited in the literature $[14,33]$ to synthesize and characterize the physical hydrogels at the micro- or nanoscale. During the synthesis process in this study, the heated aqueous mixtures of NaCMC were cooled off to produce physically cross-linked hydrogel of $\mathrm{NaCMC}$ in the form of reversible gels. After warming a mixture up to $60^{\circ} \mathrm{C}$, the polymer chains are solubilized and take the form of random coils. Upon decreasing the temperature, the chains begin to take on conformation transformation and are associated and form (double) helices (coil-helix transition). Subsequently, the double helices undergo a rearrangement in rigid helical rods by forming junction zones (sol-gel transition), and bring about the thermoreversible hydrogel $[14,17]$. These types of cellulosebased hydrogel are reversible like other physically cross-linked hydrogels. The microstructure of thermoresponsive physically crosslinked hydrogel of $\mathrm{NaCMC}$, based on coil-helix transition and forming junction zones, is shown in Figure 5.

These produced hydrogels, which are highly responsive to changes in temperature as an external stimulus and show thermoresponsive swelling and shrinking behaviors, are types of temperature-sensitive intelligent phase-reversible hydrogels, and show a bright future in delivery systems. On the one hand, raising the temperature of the suspensions decreases the number of junction zones, which brings the weaker gels about and gradually creates the transition process that results in solution development (sol state). On the other hand, cooling off the suspensions, the junction zones become more stable and therefore the stronger gels are obtained, which is in agreement with the results acquired by Matricardi et al.[34]. 
Citation: Nasri M, Mirshekarpour H (2015) Polymeric Nanostructures as Colloidal Drug Delivery Systems: Thermosensitive Hydrogels Containing Self-Assembled Micelles. J Nanomed Nanotechnol 6: 301. doi:10.4172/2157-7439.1000301

In addition to regular beneficial effects of hydrogel relating to sustained or controlled release delivery and solute permeability $[4,15]$, $\mathrm{NaCMC}$ hydrogel as a type of intelligent (smart) hydrogel looks very promising in drug delivery and shows some distinctive characteristics, including compatibility with many loaded chemicals [14,27]. It was found [35] that release of anionic and non-ionic loaded drugs from polyanionic NaCMC matrix is usual, but it is significantly extended for cationic drugs because of chemical bonds between these drugs and carboxyl groups in the dissolution medium. Therefore, this type of hydrogel can be used for designing novel prolonged release matrices for cationic chemicals. Raising the concentration of $\mathrm{NaCMC}$ during the time of formulation results in enhanced viscosity, degradation, swelling, and porosity of composed gels, but, conversely, induces decrease in stiffness, shear modulus and viscosity of the gels [36]. Furthermore, those types of carriers with a lower proportion of NaCMC in their matrix such as $\mathrm{I}_{2}$ and $\mathrm{III}_{3}$ provide faster chemical release; in an opposite manner, sustained chemical release can be obtained by employing those types composed of its higher ratios (Tables 1 and 2).

One outstanding restriction on application of hydrogel is the lack of mechanical strength, and thus we incorporated polysorbates, including a hydrophobic component, into the hydrogel to improve its mechanical strength $[37,38]$. A schematic of amphipathic polysorbate molecule is shown in Figures 6A and 6B. Polysorbates also provide the

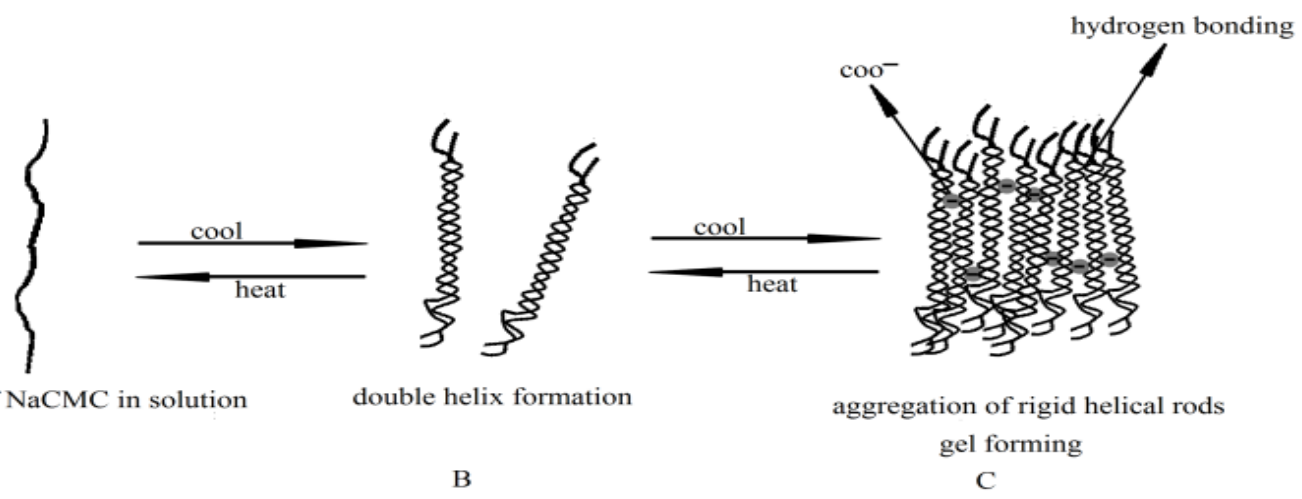

Figure 5: The basic scheme of developing the physical cross-linked hydrogel of NaCMC (sodium carboxymethylcellulose) in the form of reversible gel by heatingcooling a polymer solution. (A) Random coils of the polymer chains after warming up the mixture; (B) (double) Helixes formation (coil-helix transition) upon decreasing the temperature, (C) A rearrangement in rigid helical rods by forming junction zones (hydrogen bonding). Electrostatic repulsion between the carboxyl groups of NaCMC results in an elevated level of gel elasticity.

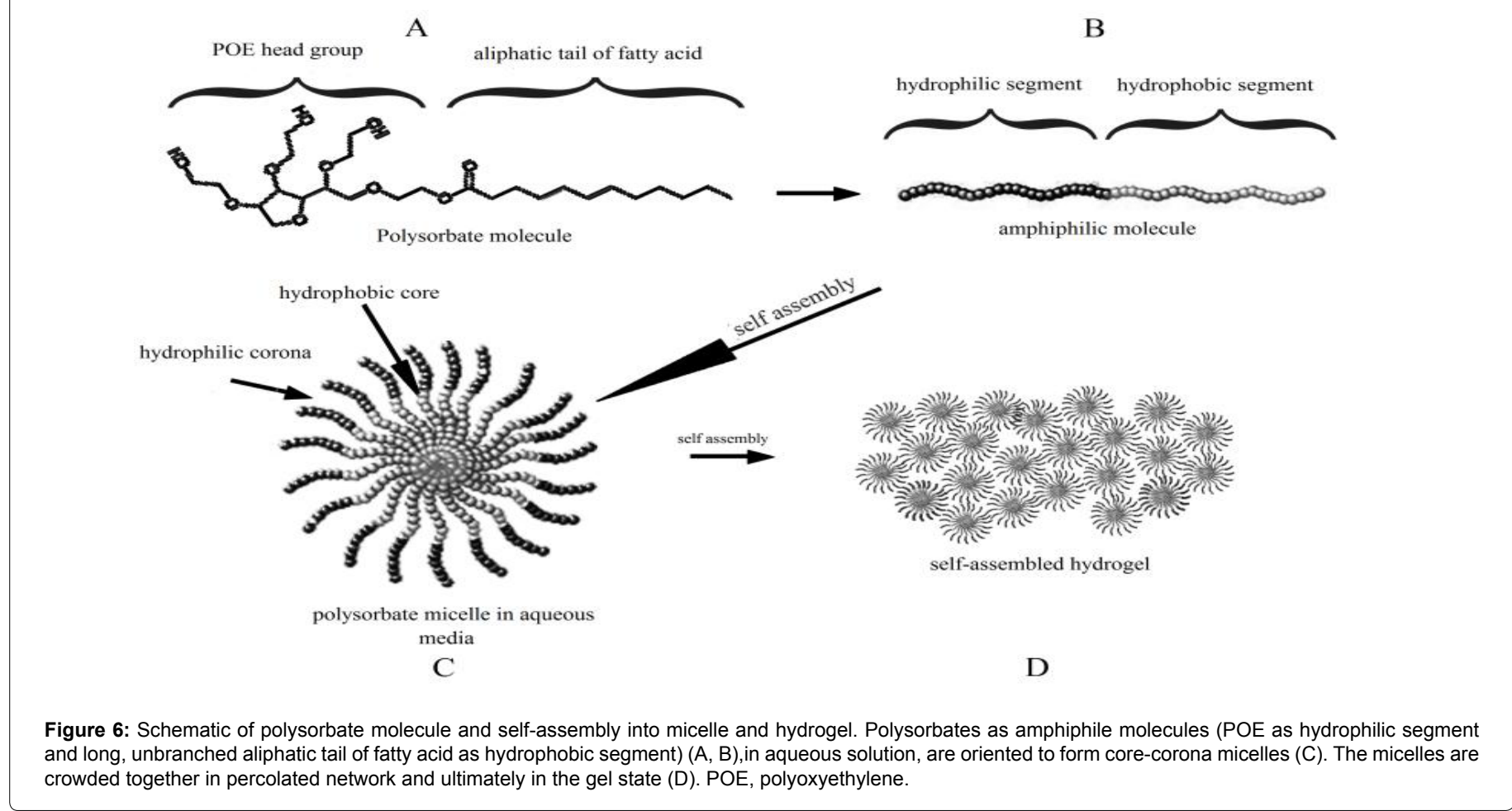


delivery system with lipophilic moiety, which can prepare sustained release of chemicals [39]. Additionally, this lipophilic segment can protect the drugs from metabolism and prolong their retention time within the delivery site [4]. It is known [38] that polysorbates, as nonionic surfactants, adsorb to the NaCMC molecules in solution and form polymer complex. Bonding between the polyoxyethylene (POE) segment of polysorbates and the $\mathrm{NaCMC}$ hydrogel improves biocompatibility and biodistribution of the gel matrix and reduces the initial burst effect $[24,35]$.

Formulating this delivery system, there has been a plan to take great advantage of unvanquishable beneficial characteristics of micellar systems $[1,3,4,7,12,13,21]$ as powerful nanocarriers in these supramolecular drug delivery systems. It is well known [40-42] that polysorbates, like other amphiphile molecules, in aqueous solution, are self-organized and oriented to form association colloids and core-corona micelles as the most thermodynamically stable state (Figure 6C). On the other hand, it is perfectly obvious that employed concentrations of each type of polysorbates throughout this study are far above its CMC (critical micelle concentration) amount [43,44], and thus it seems reasonable to conclude that the majority of polysorbate molecules in these matrixes take the form of micelles. Micelles, which are types of rarely extravasating long-circulating microparticulates, can be used as passive targeting systems for pathological sites with leaky vasculature involving tumors, inflammations, and infarct areas [21]. Forming polysorbates micelles, POE head groups orient outward and in the direction of the aqueous environment and form a corona (outer shell). And long unbranched aliphatic tails of fatty acid molecules, avoiding the water phase, associate at the center and constitute a core (inner shell) (Figure 6C). Then this corona protects the core through steric stabilization and prevents the absorption of proteins $[1,3,4,35]$. It has therefore been considered as a biocompatible polymeric nanocarrier shell. These sterically stabilized micelles develop the ability to avoid macrophages and the mononuclear phagocyte system (stealth strategy) [45], and therefore the circulation time of the delivery systems is prolonged. Therefore, these stealth micelles are regarded as longcirculating drug delivery systems. However, this steric barrier increases leakage of the loaded drugs.

Free of $\mathrm{NaCMC}$, the aqueous mixture of polysorbates also forms a gel through the course of action during which the polysorbates initially self-assemble into flowable micelles, and then the micelles are crowded together in percolated networks and ultimately in the gel state $[20,46]$. The microstructure of such self-assembled gels, which consisted of ordered polysorbate micelles, is shown in Figure 6D. As a direct result, in addition to the polymeric chains of $\mathrm{NaCMC}$, a skeletal frame of the produced hydrogels is composed of a large-scale self-assembly of micellar structures of polysorbates. It was found [20] that after gelation, the micellar structures survived virtually intact. In the opinion of Lin et al., [20,46] coarsening and increasing the size of micellar clusters (mesh size) longer than the wavelength of visible light was explained as the main reason why the gel becomes opaque. In agreement with them, it is hypothesized in this study that the produced gels become opaque or transparent on account of roughness and the size of the micelle clusters and network. Since micelle particulates and their networks are formed by aggregation of polysorbates, and precisely because all of the opaque gels were produced in steps II and III of formulation in which the concentration of polysorbates was augmented dramatically, it seems reasonable to assume that this sharp increase in polysorbates concentrations is one of the main reasons behind opaqueness of the gels. However, for the transparent gels, including all the produced types in step I, the amount of polysorbates in their compositions was several times lower than the products of steps II and III. From this evidence, it can be reasonably inferred that the degree of opaqueness and transparency of the resultant gel is directly related to the concentration of polysorbates. Some schematics of the percolated micelles network and size and roughness of the micelle clusters in transparent and opaque gels are presented in Figure 7.

As was evident, the polymeric mixture, through a repeated freezing-thawing technique, develops into a strong, flexible, and stable hydrogel structure which assembled by physical cross-linking. Urushizaki et al., found [47] that an increase in the freezing and thawing times augments the number of physical cross-linking points, and also additionally causes arising of the chains entanglement and aggregation, and an enhancement of the density. The different samples of these hydrogels take three certain and well-defined states including sol, gel, and precipitate (Figures 1 and 7). The gels are weak or strong depending on their flow behavior in the steady state, and are presented in opaque and transparent forms. The temperature reversibility of these hydrogels is clearly evident from both test-tube inverting experiments and rheological studies, in which these gels undergo sol-gel phase transformation depending on changes in temperature. However, these hydrogels show the opposite gelation property to the defining characteristics of customary thermally reversible gels $[3,20]$. It seems likely that freezing causes an increase in the close association and compactness of polysorbates that is followed by micelle formation and gelation as a direct consequence of the micelles packing (Figures $6 \mathrm{D}$, 7B and 7C). Alternatively, thawing out deconcentrates the percolated micelles and aggregated polysorbates, which results in a transition to the sol phase (Figure 7A for a schematic). With a further increase in hydrophobicity, which happened specifically following a constant rise in temperature, the micellar systems could not survive intact and are broken and destroyed, leading to a macroscopic precipitate state (Figures 1D and 7D). It can therefore be concluded that the sol-gel phase transition in these types of thermoreversible hydrogels, in addition to the temperature, is directly related to the concentration and compactness of polysorbates.

According to the literature $[7,18,32]$, the produced hydrogels, which formed through physical self-assembly, show weaker mechanical characteristics than the chemical cross-linked ones. On the other hand, physical hydrogels have been marked by ever increasing importance in the pharmaceutical applications [14,15,27], mainly due to easy production without a need for a cross-linker. Also, drugs can be loaded into them fast and in large amounts compared to the chemically crosslinked gels. Therefore, they prepare a suitable depot for incorporating drugs. The produced systems, with a lack of chemical cross-link bonds, can then also yield a faster delivery rate of drugs. However, the micelle self-assemblies show slow release kinetics [7].

The produced hydrogels reach equilibrium swelling with the change of their compositions. For some types of these produced mixtures such as $\mathrm{I}_{2}$ and $\mathrm{I}_{3}$, at the end of the swelling process, hydrogel macromolecular chains occupy the entire space of the mixtures due to the lack of crosslink bond in these types. Thus, these produced systems are characterized as liquid-like physical gel matrices possessing weak structures. For $I_{1}$ as a diluted type, a weak watery suspension is obtained. Some types such as III ${ }_{1}$ and III $_{5-10}$, including infinite polymer network with high physical cross-link density, exhibit the pseudo-swelling behavior, making the transition to the polymer network (hydrogel).

Utilizing glycerol and slowly stirring water into the slurry of $\mathrm{NaCMC}$ and glycerol induce a liquid-liquid phase separation process and cause greater swelling than the other method of preparation. At 


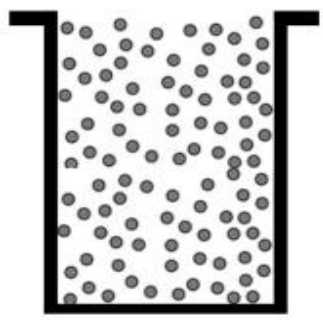

A sol

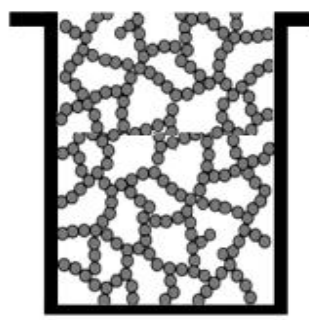

B transparent gel

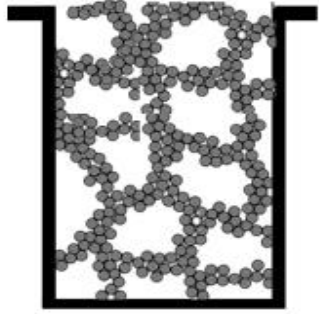

C opaque gel

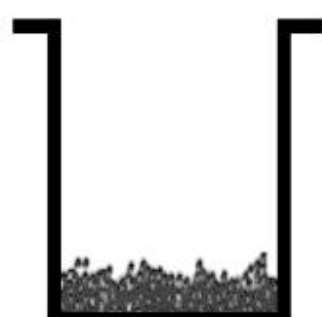

D precipitate

Figure 7: The simplified schematics of three states of temperature-sensitive phase-reversible hydrogels including sol, gel (transparent and opaque), and precipitate. Schematically, a circle represents a core-corona micelle. (A) Sol as a fluid colloidal suspension included sparse micelles at the room temperature, (B) Transparent gel as a result of an increase in concentration of polysorbates and compactness of the micelles and consequently mesh like micelles network formation, (C) At high concentrations of polysorbates, because of coarsening and increasing the size of micelles network longer than wavelength of visible light, the gel becomes opaque, (D) A macroscopic precipitate state resulted from breaking and destroying the micelless.

the time of formulation, due consideration was given to the bonding capacity between ingredients. It was revealed [38] that polysorbates molecules, after addition to the gum, attach onto the NaCMC polymer, and further addition of the polysorbates causes micelle formation on the NaCMC molecules. From a microstructure standpoint, bonding between NaCMC and POE blocks of polysorbates results in the gel with microstructure consisting of a polymer network with the micellar structures sheltered inside. It was established [40] that aqueous solution of glycerol promotes the micellization of polysorbates, and gives stability to the micelles. It is known [7] that structural stability of micellar particles systemically is such a decisive factor in the success of delivery. Accordingly, combination of these essential ingredients can form polymer complexes in interpolymer or intrapolymer types. The interpolymer complexes resulting from combinations of non-ionic (polysorbates) and ionic ( $\mathrm{NaCMC})$ polymers produce hydrophilic matrices for near zero-order release. The drug release rate in these systems can be controlled using two different approaches: polymer matrix and reservoir system. Polysorbate micelles, as a reservoir delivery system, can be drug-enriched cores with bioactive agents physically entrapped in them and encapsulated within a membrane of polymeric hydrogel. The NaCMC hydrogel can be augmented homogenously with solid particles of the drugs, and thus its network performs as a uniform matrix device into which the drugs can be physically loaded. The drug will be either dispersed or dissolved in the sol state. The suspension technique is generally employed for water insoluble drugs, but for drugs of other solubility states, micelles as the particulate system may offer comparative advantage.

Some of these produced polymeric suspension systems, such as type III $_{10}$ and the product of five freezing-thawing cycles, transformed gradually into a ternary system consisted of three phases (Figure 4). One of these phases comprises the swollen gel fraction made up of a partially cross-linked polymers network, which is suspended in the denser part of the bulk of suspension. The other phase is a high molecular weight solvent with a seemingly murkier part of the suspension placed above the clearer part which consists of finite unattached polymer chains (sol fraction) diffused out of an infinite branched network (gel fraction) into the solvents during the swelling of the gel. And the third phase involves a low molecular weight solvent seemingly like a looser part of the suspension, which is located at a lower part of it. Figure 8 shows a schematic of such ternary systems included three phases: (i) a swollen gel fraction, (ii) a high molecular weight solvent, and (iii) swollen gel

infinite branched polymer network

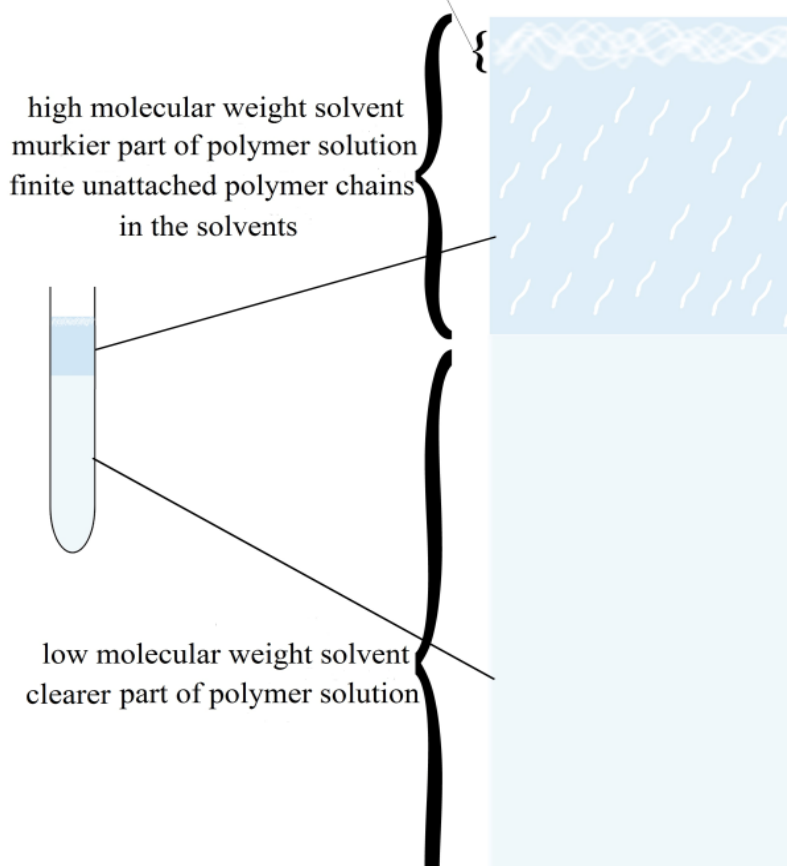

Figure 8: Schematic representation of a produced polymeric suspension involved a ternary system including: a swollen gel fraction as a partially cross-linked polymers network suspended in the denser part of bulk of suspension; a high molecular weight solvent as a murkier part of the suspension placed above the clearer part; and a low molecular weight solvent as a clearer part located at a lower section of the suspension.

a low molecular weight solvent. Introduction of glycerol as a nonsolvent induces ordered agglomerates to form, which mostly have the appearance of dendrites, in the polymer solution (in the schematic of Figure 8 is illustrated as the infinite branched polymer network). The polymeric agglomerates in the gel state mainly exist in a layer at the top 
Citation: Nasri M, Mirshekarpour H (2015) Polymeric Nanostructures as Colloidal Drug Delivery Systems: Thermosensitive Hydrogels Containing Self-Assembled Micelles. J Nanomed Nanotechnol 6: 301. doi:10.4172/2157-7439.1000301

of the colloidal suspensions after solutions make the transition to gels, and also in the sol state suspended at the top of the medium.

This formulation was developed to provide prolonged mucosal adhesion and sustained delivery of a drug through the mucosal membrane. As a new and different approach to extend the residence time of dosage forms on the mucosa, $\mathrm{NaCMC}$ was incorporated in the mixtures to augment the viscosity of the delivery systems. NaCMC, additionally, was employed on account of its mucoadhesion property (as represented in Figure 9 schematically) $[4,15]$. The physically selfassembled hydrogels regularly hold mucoadhesion characteristics in more strength compared to chemical types of hydrogel, simply because cross-link bonds reduce chain flexibility and bring about a large decrease in mucoadhesion. It is known [4] that mucoadhesive micelles show the same mechanisms as bioadhesive powder in the way that they absorb water and form a gel-like structure. For drug delivery, these mucoadhesive micelle-like compositions can be formulated in the form of suspension, aerosol, dry powder, or (semi)solid forms such as gel and cream. On the other hand, as it was reported [4], a combination of $\mathrm{NaCMC}$ as mucoadhesive and polysorbates as nonmucoadhesive polymers is a systematic way of optimizing bioadhesive characteristic of the carriers. The essential ingredients possess known properties of mucosal permeation enhancer: 1.NaCMC is recognized as a safe penetration enhancer for hydrophilic compounds; 2.polysorbates, especially polysorbates 80 and 20; and 3.fatty acid tails of polysorbates [2-4]. However, there are potential harmful effects in utilizing penetration enhancers which may be originated directly from disturbing cell structures/functions or indirectly from enhancing penetration of toxic agents and organisms.

\section{Conclusion}

This paper reports formulation, design, development, and characterization of a new group of colloidal suspensions based on hydrogel-nanoparticles. The novel products offer considerable future potential to become nanostructure-mediated drug delivery and release systems mainly because the essential ingredients and also their compositions are almost biocompatible, non-toxic and nonimmunogenic, and biodegradable. Additionally, these delivery systems provide a desired loading capacity with various types of chemicals with different physicochemical and surface properties due largely to pharmaceutical characteristics of NaCMC physically cross-linked hydrogel and stealth polysorbates micelles. These polymeric matrices, which contained POE, are crucially important to maintaining integrity of the matrices and providing long circulating and extended drug release. The novel delivery systems also minimize leakage during the

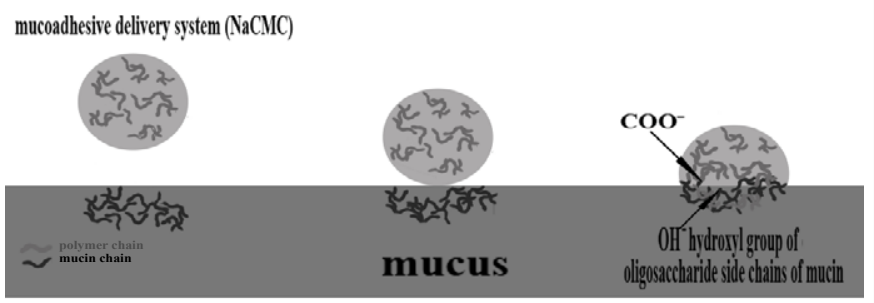

Figure 9: Schematic of NaCMC mucoadhesive hydrogel on the mucosa. (Middle) In contact stage, an intimate contact between mucoadhesive gel matrix and mucus is formed. (Right) In consolidation stage, NaCMC polymer chains interpenetrate with mucus glycoprotein, carboxyl groups of $\mathrm{NaCMC}$ can bond with hydroxyl end groups of oligosaccharide side chains of mucus protein, providing a prolonged adhesion. distribution, and protect sensitive chemical molecules. However, these physically self-assembled hydrogels show the opposite gelation property to conventional thermoresponsive hydrogels in biomedical applications. We hypothesize in this study that the concentration of polysorbates is one of the main reasons behind gelation of the mixtures and also opaqueness of the produced gels. These new delivery systems would be of great value to mucosal delivery regarding mucosal penetration enhancers and mucoadhesion property.

\section{Acknowledgment}

This work was supported by the Nuclear Medicine Department of Shafah Hospital, Kerman University of Medical Sciences. The authors acknowledge the School of Pharmacy as well as the Afzalipour School of Medicine, Kerman University of Medical Sciences, for partial support.

\section{References}

1. Gourley DR, Eoff III JC (2010) The APhA Complete Review for Pharmacy.

2. Swarbrick J, Boylan JC (2007) Encyclopedia of pharmaceutical technology.

3. Hillery AM, Lloyd AW, Swarbrick J (2005) Drug Delivery and Targeting for Pharmacists and Pharmaceutical Scientists. Taylor \& Francis, New York, USA

4. Dumitriu S (2002) Polymeric biomaterials. $2^{\text {nd }}$ edtn, CRC Press, New York USA.

5. Discher DE, Ahmed F (2006) Polymersomes. Annu Rev Biomed Eng 8: 323 341.

6. Pillai O, Panchagnula R (2001) Polymers in drug delivery. Curr Opin Chem Biol 5: 447-451.

7. Yang L, Alexandridis $P$ (2000) Physicochemical aspects of drug delivery and release from polymer-based colloids. Current Opinion in Colloid and Interface Science 5: 132-143.

8. Hughes GA (2005) Nanostructure-mediated drug delivery. Nanomedicine 1 : 22-30.

9. Koo OM, Rubinstein I, Onyuksel H (2005) Role of nanotechnology in targeted drug delivery and imaging: a concise review. Nanomedicine 1: 193-212.

10. Davis SS (1997) Biomedical applications of nanotechnology--implications for drug targeting and gene therapy. Trends Biotechnol 15: 217-224.

11. Singh R, Lillard JW Jr (2009) Nanoparticle-based targeted drug delivery. Exp Mol Pathol 86: 215-223.

12. Haag R (2004) Supramolecular drug-delivery systems based on polymeric core-shell architectures. Angew Chem Int Ed Engl 43: 278-282.

13. Rangel-Yagui CO, Pessoa A Jr, Tavares LC (2005) Micellar solubilization of drugs. J Pharm Pharm Sci 8: 147-165.

14. Gulrez SKH, Al-Assaf S (2011) Hydrogels: Methods of Preparation Characterisation and Applications. Progress in Molecular and Environmental Bioengineering - From Analysis and Modeling to Technology Applications.

15. Pal K, Banthia AK, Majumdar DK (2009) Polymeric Hydrogels: Characterization and Biomedical Applications-A mini review. Designed Monomers and Polymers 12: $197-220$

16. Omidian H, Park K (2008) Swelling agents and devices in oral drug delivery. Journal Of Drug Delivery Science And Technology 18: 83-93.

17. Schacht EH (2004) Polymer chemistry and hydrogel systems. Journal of Physics: Conference Series 3: 22

18. Chen H, Fan M (2008) Novel Thermally Sensitive pH-dependent Chitosan Carboxymethyl Cellulose Hydrogels. Journal of Bioactive and Compatible Polymers 23: 38-48.

19. Moghadam MN, Kolesov V, Vogel A, Klok HA, Pioletti DP (2014) Controlled release from a mechanically-stimulated thermosensitive self-heating composite hydrogel. Biomaterials 35: 450-455.

20. Yu L, Chang G, Zhang H, Ding J (2007) Temperature-induced spontaneous sol-gel transitions of poly(D,L-lactic acid-co-glycolic acid)-b-poly(ethylene glycol)-b-poly(D,L-lactic acid-co-glycolic acid) triblock copolymers and their end-capped derivatives in water. Journal of Polymer Science Part A: Polymer Chemistry 45: 1122-1133. 
Citation: Nasri M, Mirshekarpour H (2015) Polymeric Nanostructures as Colloidal Drug Delivery Systems: Thermosensitive Hydrogels Containing Self-Assembled Micelles. J Nanomed Nanotechnol 6: 301. doi:10.4172/2157-7439.1000301

21. Torchilin VP (2001) Structure and design of polymeric surfactant-based drug delivery systems. J Control Release 73: 137-172.

22. Chatterji PR, Kaur H (1992) Interpenetrating hydrogel networks: 3. Properties of the gelatin-sodium carboxymethylcellulose system. Polymer 33: 2388-2391.

23. Conti S, Maggi L, Segale L, Ochoa Machiste E, Conte U, et al. (2007) Matrices containing NaCMC and HPMC 2. Swelling and release mechanism study. Int J Pharm 333: 143-151.

24. Kumari A, Yadav SK, Yadav SC (2010) Biodegradable polymeric nanoparticles based drug delivery systems. Colloids Surf B Biointerfaces 75: 1-18.

25. Nasri M, Mirshekarpour $\mathrm{H}$ (2009) Targeting the pulmonary delivery via optimizing physicochemical characteristics of instilled liquid and exploring distribution of produced liquids by bench-top models and scintigraphy of rabbits' lungs. World Academy of Science, Engineering and Technology 56: 701-711.

26. Nie H, Liu M, Zhan F, Guo M (2004) Factors on the preparation of carboxymethylcellulose hydrogel and its degradation behavior in soil. Carbohydrate Polymers 58: 185-189.

27. Sannino A, Demitri C, Madaghiele M (2009) Biodegradable Cellulose-based Hydrogels: Design and Applications. Materials 2: 353-373.

28. Brandner JD (1998) The composition of NF-defined emulsifiers: sorbitan monolaurate, monopalmitate, monostearate, monooleate, polysorbate 20 , polysorbate 40, polysorbate 60, and polysorbate 80 . Drug Dev Ind Pharm 24 1049-1054.

29. Amidon G, Peck G, Block L, Moreton R, Katdare A, et al. (2007) Excipient Performance. Pharmacopeial Forum 33.

30. Rowe RC, Sheskey PJ, Quinn ME (2009) Handbook of Pharmaceutical Excipients. $9^{\text {th }}$ edtn, Pharmaceutical Press and American Pharmacists Association, UK.

31. Hoefler A-Sodium Carboxymethyl Cellulose Chemistry, Functionality, and Applications. Food Ingredients Group, Hercules Incorporated. Food Ingredients Group, Hercules Incorporated, Wilmington, Delaware 19808.

32. Barbucci R, Magnani A, Consumi M (2000) Swelling Behavior of Carboxymethylcellulose Hydrogels in Relation to Cross-Linking, $\mathrm{pH}$, and Charge Density. Macromolecules 33: 7475-7480.

33. Mahmoud Nasef M, El-Hefian EA, Saalah S, Yahaya AH (2011) Preparation and Properties of Non-Crosslinked and Ionically Crosslinked Chitosan/Agar Blended Hydrogel Films. E-Journal of Chemistry 8: S409-S419.

34. Matricardi P, Cencetti C, Ria R, Alhaique F, Coviello T (2009) Preparation and characterization of novel gellan gum hydrogels suitable for modified drug release. Molecules 14: 3376-3391.

35. Palmer D, Levina M, Nokhodchi A, Douroumis D, Farrell T, et al. (2011) The influence of sodium carboxymethylcellulose on drug release from polyethylene oxide extended release matrices. AAPS PharmSciTech 12: 862-871.

36. Lombardo L, Reeves R, Ribeiro A, Leach JB (2007) Crosslinked Carboxymethylcellulose Hydrogels: Versatile Platforms for Studying Cellular Behavior in 3D Biomaterials.

37. Michailova V, Titeva S, Kotsilkova R, Krusteva E, Minkov E (2000) Water uptake and relaxation processes in mixed unlimited swelling hydrogels. Int $\mathrm{J}$ Pharm 209: 45-56.

38. Sovilj V, Dokic P, Petrovic L (2003) Rheological Properties Of Sodium Carboxymethylcellulose In The Presence Of Electrolyte And Mixed Micelle Of Surfactants. Acta periodica technologica: 7 1-82.

39. Fu J, Fiegel J, Krauland E, Hanes J (2002) New polymeric carriers for controlled drug delivery following inhalation or injection. Biomaterials 23: 4425-4433.

40. Aizawa H (2009) Morphology of polysorbate 80 (Tween 80) micelles in aqueous, 4-dioxane solutions. Journal of Applied Crystallography 42: 592-596.

41. Deechongkit S, Wen J, Narhi LO, Jiang Y, Park SS, et al. (2009) Physical and biophysical effects of polysorbate 20 and 80 on darbepoetin alfa. J Pharm Sci 98: 3200-3217.

42. Kerwin BA (2008) Polysorbates 20 and 80 used in the formulation of protein biotherapeutics: structure and degradation pathways. J Pharm Sci 97: 2924 2935.

43. Patist A, Bhagwat $S$, Penfield $K$, Aikens $P$, Shah $D$ (2000) On the measurement of critical micelle concentrations of pure and technical-grade nonionic surfactants. Journal of Surfactants and Detergents 3: 53-58.

44. Wan LS, Lee PF (1974) CMC of polysorbates. J Pharm Sci 63: 136-137.

45. Storm G, Belliot SO, Daemen T, Lasic DD (1995) Surface modification of nanoparticles to oppose uptake by the mononuclear phagocyte system. Advanced Drug Delivery Reviews 17: 31-48.

46. Yu L, Zhang Z, Ding J (2012) In vitro degradation and protein release of transparent and opaque physical hydrogels of block copolymers at body temperature. Macromol Res 20: 234-243.

47. Stauffer SR, Peppast NA (1992) Poly(vinyl alcohol) hydrogels prepared by freezing-thawing cyclic processing. Polymer 33: 3932-3936. 